\title{
Role of aquaporin-4 in the regulation of migration and invasion of human glioma cells
}

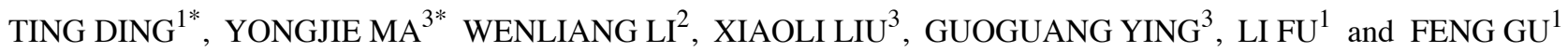 \\ Departments of ${ }^{1}$ Breast Pathology, ${ }^{2}$ Neurosurgery, and ${ }^{3}$ Central Laboratory of the Oncology Department, Key Laboratory \\ of Breast Cancer Prevention and Therapy of the Ministry of Education; Key Laboratory of Cancer Prevention and \\ Therapy of Tianjin, Tianjin Medical University, Cancer Institute and Hospital, Tianjin 300060, P.R. China
}

Received December 1, 2010; Accepted January 11, 2011

DOI: $10.3892 /$ ijo.2011.983

\begin{abstract}
Glioblastoma is the most aggressive form of primary brain tumor with a tendency to invade surrounding healthy brain tissues, rendering tumors of this type largely incurable. Aquaporin-4 (AQP4) is a key molecule involved in maintaining water and ion homeostasis in the central nervous system and has been recently reported to play a role in cell migration in addition to its well-known function in brain edema. Increased AQP4 expression has been demonstrated in glioblastoma multiforme (GBM), suggesting that it is also involved in malignant brain tumors. Here, we identify a novel role for aquaporin-4 in glioblastoma cell migration and invasion. In the present study, we used small-interference RNA technology and a pharmacological inhibitor to knock down the expression of AQP4, which resulted in specific and massive impairment of glioblastoma cell migration and invasion in vitro and in vivo. In addition, we demonstrated the possible mechanisms by which AQP4 functions in the process of glioblastoma cell invasion. The downregulation of matrix metalloprotease-2 (MMP-2) expression in LN229 cells with AQP4 reduction coincided with decreased cell invasive ability. Furthermore, our study showed that AQP4 may also be involved in the regulation of glioblastoma cell adhesion. The expression of $\beta$-catenin and connexin 43 were increased in AQP4-downregulated LN229 cells consistent with their enhanced cell-cell adhesion ability. In summary, our results indicate that AQP4 is involved in the control of glioblastoma cell migration and invasion and may be a potential therapeutic target for glioblastoma cell infiltration.
\end{abstract}

Correspondence to: Dr Feng Gu, Department of Breast Pathology, Tianjin Medical University, Cancer Institute and Hospital, Huanhuxi Road, Hexi, Tianjin 300060, P.R. China

E-mail: yongjiemagu@yahoo.com.cn

*Contributed equally

Key words: glioblastoma, AQP4, adhesion, migration

\section{Introduction}

Glioblastomas are the most common malignant tumors of the adult central nervous system. These are highly invasive and infiltrative tumors which are associated with a poor prognosis and median patient survival of only one year $(1,2)$. A major barrier to available malignant glioma treatment is the invasion of these cells into brain parenchyma. Because of this, local therapies such as surgery or radiation therapy are ineffective (3). Glioma cells invade through the ECM of the brain by activating a number of coordinated cellular processes, which include those necessary for migration and invasion. Therefore, a detailed understanding of the mechanisms underlying this invasive behavior is essential for the development of novel effective therapies.

AQPs (aquaporins) are a family of water channel proteins that provide a major pathway for osmotically driven water transport through cell membranes. To date, 13 aquaporin isoforms (AQP0-AQP12) have been identified in mammalian species (4). Both AQP1 and AQP4 have been clearly identified in brain, and AQP4 is known to participate mainly in brain edema after injury or other brain diseases (5). AQP4 is primarily expressed at the border between brain parenchyma and major fluid compartments, including astrocyte foot processes and glia limitans, as well as ependymal cells and subependymal astrocytes (6). This distribution suggests that AQP4 controls water fluxes into and out of the brain parenchyma.

Recent studies on AQP4 are not restricted to brain edema, and various researchers have discovered a new role for AQP4 in cell migration. In an in vivo model of reactive gliosis and astroglial cell migration produced by cortical stab injury, glial scar formation was markedly impaired in AQP4-null mice, with reduced migration of reactive astroglia noted towards the site of injury (7). Previously, studies have reported that AQP4 is significantly upregulated in glioblastoma compared to low grade gliomas and normal brain tissues (8-10). These studies suggest that AQP4 may be involved in glioma malignancy.

The main aim of the present study was to test the hypothesis that AQP4 plays an important role in regulating the fundamental properties of glioblastomas in regards to migration and invasion. In the present study, glioblastoma LN229 cells, in which AQP4 was downregulated, exhibited 
significant impairment in a series of migration and invasion assays in vitro. Furthermore, our data indicated that several key proteins including MMP-2, MMP-9, connexin 43 and $\beta$-catenin, were involved in AQP4 signaling pathways which are critical for glioblastoma cell migration and invasion. The results concerning invasion in an orthotopic glioma graft model in vivo further supported our data in vitro. To conclude, our study suggests that AQP4 is required in glioblastoma cell migration and invasion, and may be a therapeutic target molecule for malignant glioma infiltration.

\section{Materials and methods}

Cell culture and reagents. Human glioblastoma cell line LN229 was obtained from the American Type Culture Collection (Manassas, VA, USA). The recombinant human epithelial growth factor (EGF) was obtained from R\&D Systems (Minneapolis, MN, USA). Chemotaxis chambers and membranes were from Neuroprobe (Gaithersburg, MD, USA). Fibronectin (0.1\%) was from Sigma (St. Louis, MO, USA) and the Alexa Fluor 568 phalloidin was from Molecular Probes (Eugene, OR, USA). Neutral red staining solution (0.1\%) was from Santa Cruz Biotechnology (Santa Cruz, CA, USA). PMA (phorbol 12-myristate 13-acetate) was from Sigma. Antibodies for $\beta$-actin and rabbit anti-AQP4 and mouse anti- $\beta$-catenin antibodies were acquired from Santa Cruz Biotechnology. Rabbit anti-MMP-2 and anti-MMP-9 Antibodies were from Cell Signaling Technology (Beverly, MA, USA). Rabbit anti-connexin 43 was purchased from Santa Cruz Biotechnology.

RNA interference. Cells were plated in a 35-mm dish for $24 \mathrm{~h}$ before transfection in complete medium. The transfection was performed with Lipofectamine 2000 (Invitrogen, Carlsbad, CA) according to the manufacturer's instructions. AQP4-specific siRNA plasmids for LN229 cells (insert: GCTCAATAGCTTTAGCAATTG and scrambled sequence inserted into pGPU6/GFP/Neo) were from GenePharma Corp. (Shanghai, China). To establish stable siAQP4 cell lines, the G418-resistant cells were screened and the expression level of AQP4 protein was monitored by Western blotting.

Western blotting. Western blotting was performed as described by Zhang et al (11). In brief, cells were lysed in 1X SDS lysis buffer (Tris-HCl, pH 6.8, $62.5 \mathrm{mM}, 2 \% \mathrm{SDS}$ and $10 \%$ glycerol) followed by centrifugation at $10,000 \mathrm{rpm}$ for $10 \mathrm{~min}$ at $4^{\circ} \mathrm{C}$. Equal amounts of cell lysates $(20-40 \mu \mathrm{g}$ total protein/ lane) were loaded and separated by SDS-PAGE, and proteins were transferred onto nitrocellulose membranes, probed with anti-AQP4 (1:1000), -MMP-2 (1:1000), -MMP-9 (1:1000), -N-cadherin (1:2000), $-\beta$-catenin (1:1000) and $-\beta$-actin (1:5000) followed by HRP-conjugated or AP-conjugated secondary antibodies. The results were visualized using enhanced chemiluminescence reagents ECL (Pierce, Rockford, IL), or the mixture of nitrotetrazolium blue chloride (NBT) and 5-bromo-4-chloro-3-indolyl phosphate p-toluidine salt (BCIP) (Santa Cruz).

Osmotic fragility test. Cells were plated in 35-mm dishes at a density of $6 \times 10^{5}$ cells $/ \mathrm{ml}$ to form a monolayer. Twenty-four hours later, the medium was replaced with double-distilled water, and the cells were incubated for $2 \mathrm{~min}$. Dead and surviving cells were stained using the Neutral red staining solution $(0.1 \%)$ as follows. The wells were washed twice with phosphate-buffered saline (PBS) and subsequently incubated with Neutral red staining solution $(0.1 \%)$ at $37^{\circ} \mathrm{C}$ in a $5 \% \mathrm{CO}_{2}$ incubator for $30 \mathrm{~min}$. The surviving cells were counted under a light microscope at x200 (12).

Chemotaxis and chemokinesis assays. The chemotaxis assay was performed in a 48-well Boyden chamber as described by Zhang et al (11) In brief, the chemoattractant (EGF) was loaded into the lower chemotaxis chamber, and $8 \times 10^{5}$ cells/ $\mathrm{ml}$ were suspended in the binding medium (RPMI-1640, $0.1 \%$ BSA and $25 \mathrm{mM}$ HEPES) and added to the upper chamber. The polycarbonate filter $(8-\mu \mathrm{m})$ was pre-treated with $10 \mu \mathrm{g} / \mathrm{ml}$ fibronectin overnight, dried in air, and inserted between the upper and lower chambers. The chamber was then incubated at $37^{\circ} \mathrm{C}$ in a $5 \% \mathrm{CO}_{2}$ incubator for $3 \mathrm{~h}$. The filter membrane was then rinsed, fixed and stained. The numbers of migrating cells were counted at $\mathrm{x} 400$ in three separate fields using light microscopy. For the chemokinesis assay (checker board assay), cells were suspended in a medium containing different concentrations of EGF before being added to the upper chamber (11). All samples were tested in triplicate, and the data are expressed as the mean \pm SD.

Scratch assay. One day before the scratch assay, cells were plated in $35-\mathrm{mm}$ dishes at a density of $8 \times 10^{5}$ cells $/ \mathrm{ml}$ to form a monolayer. Then, a wound was made with an even trace in the middle of the monolayer using a $10-\mu 1$ pipette tip. The cells were then incubated at $37^{\circ} \mathrm{C}$ in a $5 \% \mathrm{CO}_{2}$ incubator, and images of the wound were captured at intervals. The distance of the wound was measured under a light microscope. All samples were tested in triplicate, and the data are expressed as the mean $\pm \mathrm{SD}$.

Cytotoxicity assay. Cell viability was assessed by the MTT assay. Cells (7000/well) were plated in 96-well plates in $100 \mu \mathrm{l}$ of complete medium. Following overnight attachment, the cells were exposed to different concentrations of PMA (1, 5 and $10 \mu \mathrm{M})$ for 1 day. Then $10 \mu \mathrm{l}$ of 3-(4,5-dimethylthiazol2-yl)-2,5-diphenyltetrazolium bromide (MTT) solution was added to each well. After $4 \mathrm{~h}$ of incubation at $37^{\circ} \mathrm{C}$, followed by the addition of $150 \mu 1$ dimethyl sulfoxide to each well and shaking for $10 \mathrm{~min}$, the absorbance was measured at $490 \mathrm{~nm}$ by a microplate reader.

Adhesion assay. The adhesion assay was carried out as previously described (13). Cells $\left(4 \times 10^{5} / \mathrm{ml}\right)$ were suspended in medium with $10 \%$ serum and incubated at $37^{\circ} \mathrm{C}$ in a $5 \%$ $\mathrm{CO}_{2}$ incubator for $20 \mathrm{~min}$. Then, $1.5 \mathrm{ml}$ of cell solution was promptly added to a $35-\mathrm{mm}$ dish containing dried glass coverslips. The coverslips were pre-treated with $10 \mu \mathrm{g} / \mathrm{ml}$ of fibronectin in serum-free medium for $2 \mathrm{~h}$ at $37^{\circ} \mathrm{C}$ and then airdried for $30 \mathrm{~min}$ at room temperature. After 5, 15 and $30 \mathrm{~min}$ of incubation, the cells were gently washed twice with cold PBS and then fixed with $4 \%$ paraformaldehyde for $20 \mathrm{~min}$. The cells attached to the coverslips were counted under a light microscope at $\times 200$. 
Detachment assay. The cellular detachment assay was carried out as described previously (14). Briefly, the cells were seeded at $2 \times 10^{5}$ cells $/ 35-\mathrm{cm}^{2}$ dish. The dishes were pre-treated with $1.2 \mathrm{mg} / \mathrm{ml}$ of Matrigel. After $48 \mathrm{~h}$, the cells were washed with $5 \mathrm{ml}$ of warm PBS and then trypsinized with $1 \mathrm{ml}$ of fresh $0.25 \%$ trypsin at $20^{\circ} \mathrm{C}$ with gentle agitation. The number of detached cells was determined at various time points, and the total number of cells/dish was determined after complete trypsinization. One dish was used for each time point, and each experiment was performed at least five times independently.

Aggregation assay. Cell-cell adhesion ability was detected by an aggregation assay, which was carried out as described previously (15). Cells were washed three times with RPMI1640 (serum-free medium) and were subsequently trypsinized with $4.0 \mathrm{mM}$ EDTA with calcium to protect cadherin subtypes on the cell surface from cleavage. After centrifugation, the cells were resuspended in RPMI-1640 (serum-free medium). A 2-ml volume of single-cell suspension at a concentration of $5 \times 10^{5} / \mathrm{ml}$ from each group was poured into $1 \%$ BSA-coated $35-\mathrm{mm}$ plates and incubated at $37^{\circ} \mathrm{C}$ in a $5 \% \mathrm{CO}_{2}$ incubator for different time periods. The cells and cell clusters were counted using a hemocytometer after incubation for 15, 30 and $45 \mathrm{~min}$ and $1 \mathrm{~h}$. The aggregation index was calculated using the formula below and inversely correlates with cell aggregation. Aggregation index $(\mathrm{AI})=$ (Number of single cells + Number of cell clusters)/Total number of cells initially added.

Cellular F-actin measurement. Cellular F-actin measurement was carried out as described previously (11). Following stimulation with $50 \mathrm{ng} / \mathrm{ml} \mathrm{EGF}$ at $37^{\circ} \mathrm{C}$ for different time periods, cells were fixed, permeablized, and incubated with Oregon Alexa Fluor 568 phalloidin in F-actin buffer $(10 \mathrm{mmol} / \mathrm{l}$ HEPES, $20 \mathrm{mmol} / 1 \mathrm{KH}_{2} \mathrm{PO}_{4}, 5 \mathrm{mmol} / 1 \mathrm{EGTA}, 2 \mathrm{mmol} / 1$ $\mathrm{MgCl}_{2}, \mathrm{PBS}, \mathrm{pH}$ 7.4) at room temperature for $2 \mathrm{~h}$. The cells were washed five times. The labeled phalloidin that bound to F-actin was extracted using methanol at $4^{\circ} \mathrm{C}$ for $90 \mathrm{~min}$. The fluorescence was captured at Ex/Em 578/600 nm in each sample and normalized against the total protein content as analyzed using a BCA kit (Pierce, Rockford, IL, USA). The relative $\mathrm{F}$-actin content over different time periods was calculated by the following equations: F-actin $\triangle \mathrm{t} / \mathrm{F}$-actin 0 $=$ fluorescence $\Delta \mathrm{t} /$ fluorescence 0 . All samples were tested in triplicate, and data are expressed as the mean \pm SD.

Fluorescence microscopy. The cells were cultured one day prior to staining and then starved in serum-free medium for $3 \mathrm{~h}$. After stimulation with $50 \mathrm{ng} / \mathrm{ml} \mathrm{EGF}$ at $37^{\circ} \mathrm{C}$ for $1 \mathrm{~min}$, cells were fixed with $4 \%$ paraformaldehyde and permeablized with $0.1 \%$ Triton X-100 in PBS. For staining actin, the cells were then directly incubated with Alexa Fluor 568 phalloidin for $30 \mathrm{~min}$ in the dark and gently washed with PBS. The cells were then maintained in the PBS and directly visualized with a Nikon inverted fluorescent microscope (Nikon, Japan).

Matrigel invasion assay. Cell invasion in vitro was measured by assessing the invasion of cells through Matrigel-coated transwell inserts (Costar, Cambridge, MA, USA) as described
A LN229 scrLN229 clone 1 clone 2

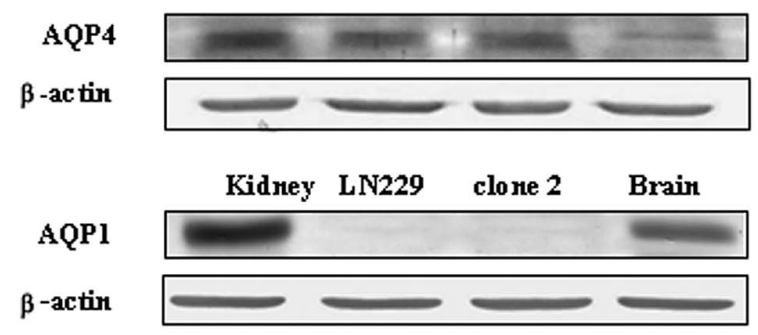

B

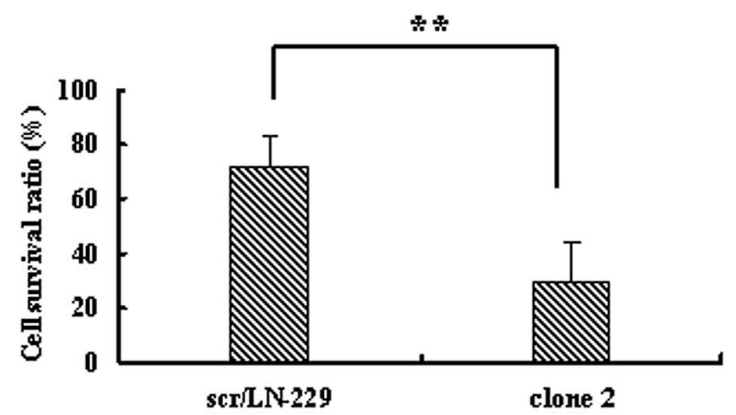

C
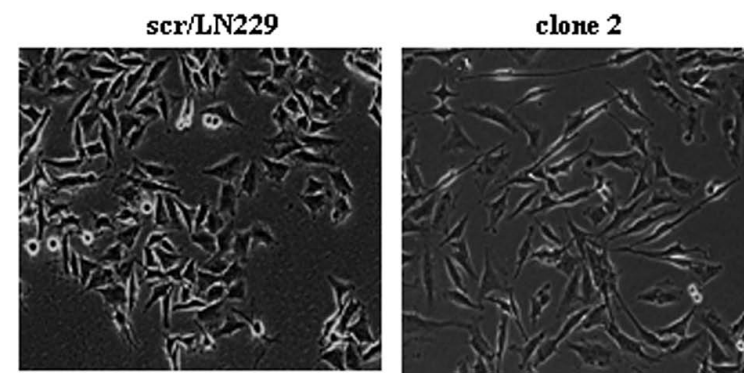

Figure 1. Establishment of stable LN229 cells expressing the AQP4 siRNA plasmid. (A) Western blot analysis of AQP4 expression in parental LN229, scr/LN229 and siAQP4/LN229 clone 1 and clone 2 cells. Expression of AQP1 in the parental LN-229 and siAQP4/LN229 clone 2 cells was also assessed; kidney and normal brain tissues were used as a positive control, and $\beta$-actin was used as a loading control. (B) Cell osmotic fragility was examined after AQP4 reduction. The survival rate of the control was $71.61 \pm 11.75 \%$ (mean \pm SD), whereas it decreased to $29.46 \pm 14.84 \%$ in the siAQP4/LN229 clone 2 cells. (C) AQP4 inhibition by siRNA caused dramatic morphological changes compared with the control cells (x200).

previously (16). Briefly, the transwell inserts $(8-\mu \mathrm{m}$ pore size) were coated with a final concentration of $3.5 \mathrm{mg} / \mathrm{ml}$ of Matrigel. The cells were trypsinized, and $200 \mu \mathrm{l}$ of cell suspension $\left(5 \times 10^{5}\right.$ cells $\left./ \mathrm{ml}\right)$ was added to triplicate wells. Binding medium (350 $\mu \mathrm{l}$ ) (RPMI-1640, 0.1\% BSA and $25 \mathrm{mM}$ HEPES) with $10 \mathrm{ng} / \mathrm{ml}$ of EGF was added to the lower well. After $24 \mathrm{~h}$ of incubation $\left(37^{\circ} \mathrm{C}, 5 \% \mathrm{CO}_{2}\right)$, the non-invading cells were removed by wiping the upper side of the membrane, and the invading cells were fixed and stained. The number of invading cells was counted under a microscope in 5 predetermined fields (total magnification, $\mathrm{x} 400$ ).

Tumor invasion in vivo. For the intracranial orthotopic human glioma model, approximately $5 \times 10^{4}$ glioblastoma cells were injected intracranially into the frontal cortex of 5-week-old male nude mice. All animals received human care according to the criteria outlined in the Guide for the Care and Use of 
A

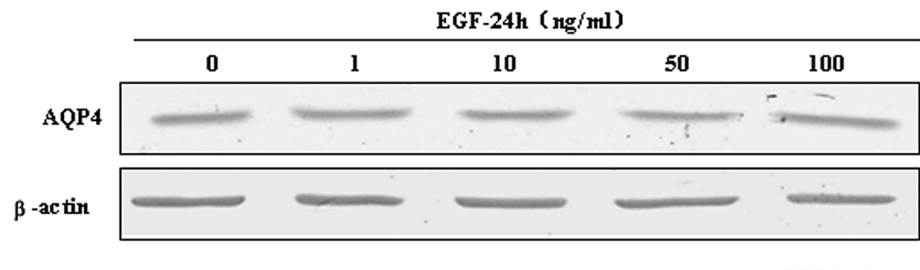

B

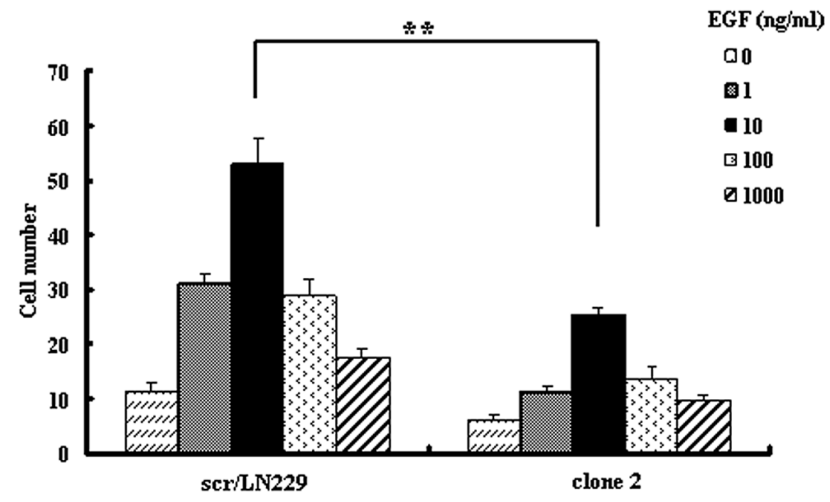

C

\begin{tabular}{|c|c|c|c|c|c|c|c|c|c|c|}
\hline EGF (ng/ml) & \multicolumn{5}{|c|}{ Upper wells (scr/LN229) } & \multicolumn{5}{c|}{ Upper wells (clome 2) } \\
\hline Lower wells & 0 & 1 & 10 & 100 & 1000 & 0 & 1 & 10 & 100 & 1000 \\
\hline 0 & $8 \pm 2$ & $13 \pm 1$ & $18 \pm 2$ & $8 \pm 1$ & $15 \pm 3$ & $6 \pm 3$ & $7 \pm 2$ & $9 \pm 1$ & $9 \pm 2$ & $14 \pm 2$ \\
\hline 1 & $19 \pm 1$ & $15 \pm 2$ & $15 \pm 3$ & $9 \pm 2$ & $10 \pm 2$ & $5 \pm 1$ & $5 \pm 2$ & $9 \pm 3$ & $11 \pm 2$ & $8 \pm 3$ \\
\hline 10 & $29 \pm 2$ & $45 \pm 2$ & $35 \pm 2$ & $12 \pm 2$ & $8 \pm 3$ & $5 \pm 2$ & $11 \pm 1$ & $10 \pm 2$ & $8 \pm 2$ & $10 \pm 2$ \\
\hline 100 & $40 \pm 3$ & $41 \pm 3$ & $42 \pm 2$ & $20 \pm 3$ & $14 \pm 3$ & $12 \pm 2$ & $9 \pm 1$ & $18 \pm 3$ & $11 \pm 3$ & $6 \pm 4$ \\
\hline 1000 & $11 \pm 2$ & $21 \pm 3$ & $27 \pm 4$ & $16 \pm 3$ & $15 \pm 3$ & $5 \pm 3$ & $13 \pm 2$ & $17 \pm 3$ & $13 \pm 3$ & $5 \pm 3$ \\
\hline
\end{tabular}

Figure 2. Disruption of AQP4 by siRNA inhibited EGF-induced chemotaxis and chemokinesis in LN229 cells. (A) Expression of AQP4 in LN229 cells was assessed after $24 \mathrm{~h}$ of EGF stimulation at different concentrations $(0,1,10,50,100 \mathrm{ng} / \mathrm{ml})$ by Western blotting. (B) Comparison of EGF-induced chemotaxis of scr/LN229 and siAQP4/LN229 clone 2 cells. A representative result from three independent experiments is shown. (Two-way ANOVA analysis, ${ }^{* *}$ p $<0.01$ ). (C) Chemokinesis assay of scr/LN229 and siAQP4/LN229 clone 2 cells.

Laboratory Animals, prepared by the National Academy of Sciences and published by the National Institutes of Health. Twenty-two mice in the control group and 26 mice in the experimental group received intracranial human glioblastoma cell transplants. Four weeks later, the mice were sacrificed, and the whole brain was fixed with $4 \%$ paraformaldehyde and removed. Samples were dehydrated, lucidied, soaked in wax, and then cut into 5-mm sections using routine histological methods.

Statistical analysis. Prism 3.0 software (San Diego, CA, USA) was used for the data analysis. The Chi-square test was used for the in vivo analysis, and the two-way ANOVA was used for two groups of data in vitro. All of the results were generated from three independent experiments.

\section{Results}

Expression of AQP4 in the human glioblastoma LN229 cell line. First, plasmids expressing AQP4 siRNA sequences were transfected into the LN229 cells to obtain stable AQP4depleted LN229 cells, which were designated as siAQP4/ LN229 cells. A siRNA vector containing a scrambled sequence was also transfected into the LN229 cells to generate control cells, which were designated as scr/LN229 cells. After the G418 selection, transfected cells were screened for AQP4 expression by Western blotting. We found that the AQP4 protein level was significantly reduced in the siAQP4/LN229 clone 2 cells compared to the control. The scr/LN229 cells displayed similar AQP4 levels as the parental LN229 cells. As expression of two aquaporins, AQP1 and AQP4, has been reported in the brain, we also assessed AQP1 expression in both LN229 and siAQP4/LN229 cells. The result showed that the expression level of AQP1 was too low to be detected in the LN229 and siAQP4/LN229 clone 2 cells; the kidney and normal brain tissues had high AQP1 expression which were regarded as the positive control (Fig. 1A).

In order to ascertain whether reduced AQP4 levels are reflected in the resistance of LN229 cells to hypo-osmotic stress, cell osmotic fragility was examined. The survival rate of the control was $71.61 \pm 11.75 \%$ (mean $\pm \mathrm{SD}$ ), whereas it decreased to $29.46 \pm 14.84 \%$ in the siAQP4/LN229 clone 2 cells (Fig. 1B). The reduced osmotic fragility of the siAQP4/LN229 clone 2 cells indicated that the AQP4 RNA interference in this clone was functional. siAQP4/LN229 clone 2 cells showed morphological changes when compared to the parental cells; the cell body of the siAQP4/LN229 cells became thin and elongated (Fig. 1C). This finding was consistent with a previously report (17). In order to confirm the function of AQP4 in LN229 cells, all subsequent functional assays and Western blot analyses were conducted using siAQP4/LN229 clone 2 cells. 
A

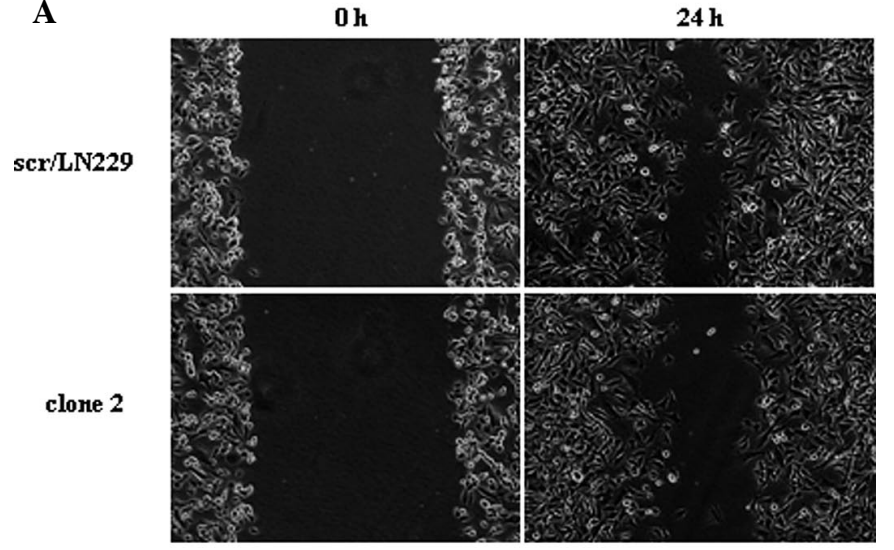

B

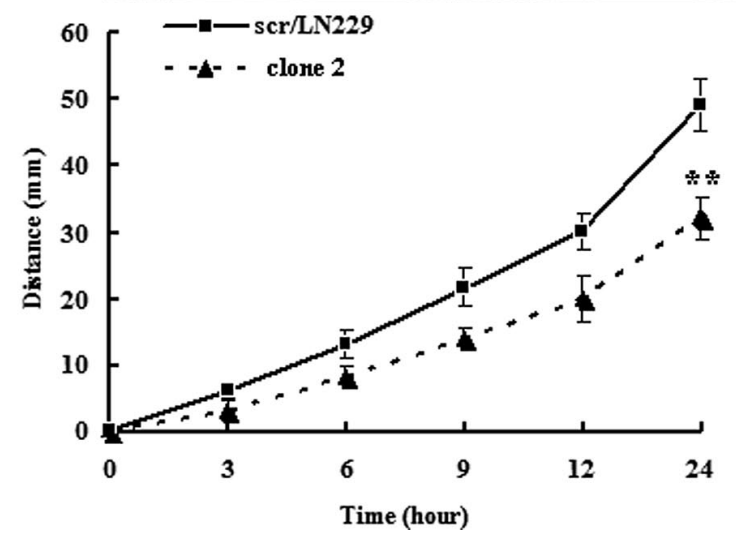

Figure 3. Scratch assay in siAQP4/LN229 clone 2 and scr/LN229 cells (A) scr/LN229 and siAQP4/LN229 clone 2 cells were grown to confluence on a $35-\mathrm{mm}$ tissue culture dish, and the monolayer was wounded with a $10-\mu 1$ pipette tip. Images were captured at 0 and $24 \mathrm{~h}$ (x100). (B) The distances of migration of the scr/LN229 and siAQP4/LN229 clone 2 cells are shown at 3 , $6,9,12,24 \mathrm{~h}$ in the scratch assay ( $(* \mathrm{p}<0.01$, two-way ANOVA analysis).

Reduction of AQP4 impairs LN229 cell migration. Migration is a fundamental property of cells that occurs during many physiological and pathological processes including organogenesis in the embryo, repair of damaged tissue after injury and the spread of cancer (18). In light of the hypothesized role of water channels in volume regulation associated with cell migration, we examined the relative role of AQP4 in cell migration.

Previous studies have shown that EGF is capable of upregulating AQP and participates in the migration of cancer cells $(19,20)$. We evaluated the expression of AQP4 in LN229 cells stimulated with different concentrations of EGF, and the result showed that AQP4 expression exhibited no distinguishable change (Fig. 2A). In order to further assess whether AQP4 is involved in EGF-induced glioma migration, we performed the following experiments. Chemotaxis refers to directional cell movement dependent on a concentration gradient. A 48-well chemotaxis model was applied to perform EGF-induced 3.5-h cell chemotaxis. The EGF induced robust chemotaxis of scr/LN229 cells, which followed a typical bell-shaped response curve. The siAQP4/LN229 clone 2 cells showed decreased chemotaxis compared with their control (Fig. 2B). Aside from chemotaxis, we also evaluated chemokinesis which presents random cell motility stimulated in a gradient-independent manner. Chemokinesis was also impaired in the siAQP4/LN229 clone 2 cells, which may have accounted for the decrease in chemotaxis (Fig. 2C). A scratch assay was previously used as an in vitro assay for wound healing and directional movement (21). When a scratch was created in the fluent cell monolayer, the siAQP4/LN229 clone 2 cells required a longer time to fill the gap (Fig. 3A and B), further supporting a defect in migration.

In addition to applying AQP4 RNA interference to reduce the AQP4 protein level, we also used the AQP4 inhibitor phorbol 12-myristate 13-acetate (PMA) which activates protein kinase $\mathrm{C}$ (PKC) and inhibits AQP4 expression (22). The results of Western blotting showed that the expression of AQP4 was significantly reduced with treatment of PMA (1 and $5 \mu \mathrm{M}$ ) compared to the control cells (Fig. 4A). As PMA treatment displayed almost no cytotoxicity to LN229 cells as shown by the MTT assay (Fig. 4B), we chose PMA at a concentration of $5 \mu \mathrm{M}$ to perform the following functional experiments. The Osmotic fragility test showed decreased osmotic fragility in PMA (5 $\mu \mathrm{M})$-treated cells compared to the control cells (Fig. 4C). To examine whether inhibition of AQP4 expression through PMA has a similar function in cell migration, we also performed cell chemotaxis and scratch assays. The chemotaxis ability of LN229 cells with PMA treatment was greatly impaired compared with the control (Fig. 4D). Similar to siAQP4/LN229 clone 2 cells, when a scratch was created in the cell monolayer, the distance of migration of the LN229 cells treated with PMA was shorter than the control cells (Fig. 4E). Taken together, our results obviously reveal that the reduction of AQP4 greatly suppresses the migratory ability of LN229 cells.

Reduction of AQP4 impairs F-actin polymerization in the LN229 cells. The formation of lamellipodia which is critical for cell migration requires extensive actin polymerization at the leading edge. The basic active mechanism involved in moving the leading edge is the polymerization of F-actin (also called actin filaments) towards the cell membrane $(23,24)$. Polymerizing F-actin alone, without any accompanying motors, can generate a significant force with which to move the leading edge of the cell. The process of F-actin polymerization is quick and transient (25). The ligand-induced transient F-actin assembly correlates with the cellular chemotactic capacity (24). The F-actin polymerization assay was performed to prove the hypothesis that the reduction of AQP4 impairs LN229 cell migration by reducing F-actin polymerization. The assay showed that the EGF elicited a transient actin polymerization at $15 \mathrm{sec}$ and $1 \mathrm{~min}$ in the control cells, consistent with a previous report (26). The immunofluorescent staining of F-actin showed that the EGF induced an increase in the F-actin content in the control cells, but not in the siAQP4/ LN229 clone 2 cells (Fig. 5B). In the siAQP4/LN229 clone 2 cells, the actin polymerization in response to EGF stimulation was significantly reduced, suggesting that AQP4 plays an important role in cytoskeleton rearrangement (Fig. 5A).

Reduction of AQP4 regulates LN229 cell adhesion. Adhesion of cells to a substrate and cell-cell adhesion are two key processes associated with cell movement (27). In the above experiment, we showed how F-actin polymerization was altered after reduction of AQP4 in LN229 cells. Adhesion 


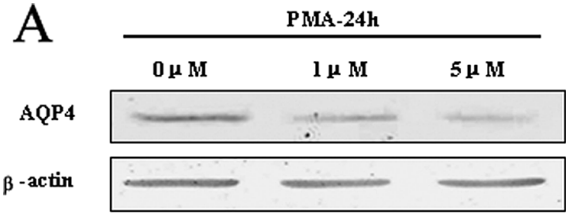

$\mathrm{B}$

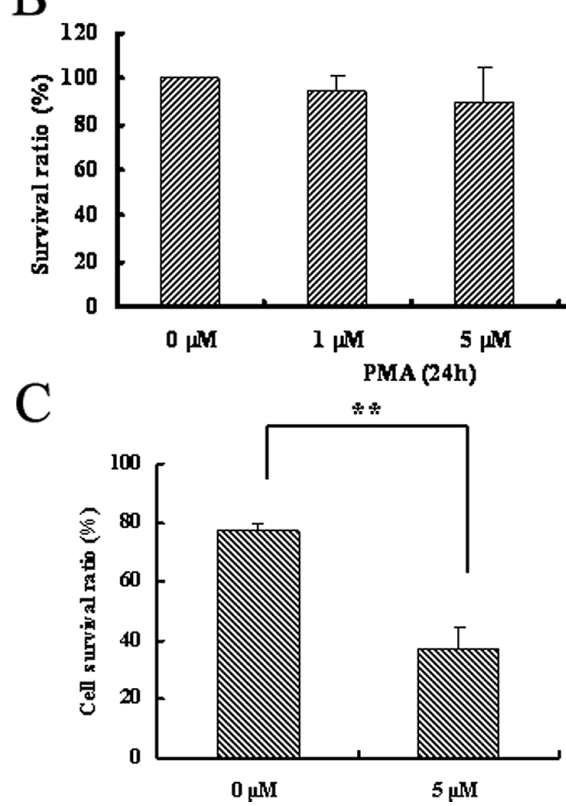

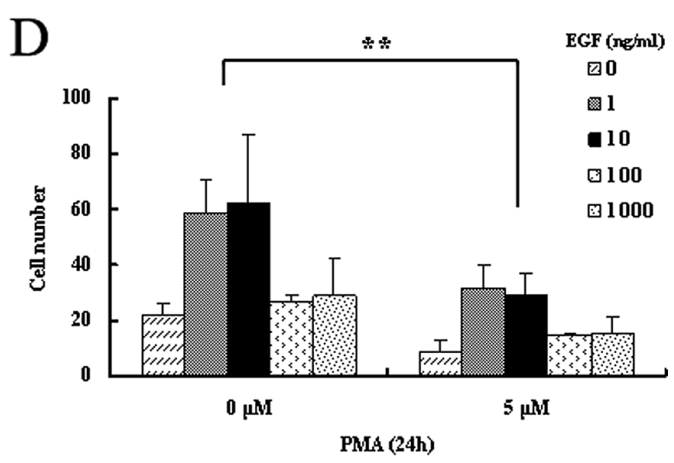

$\mathrm{E}$

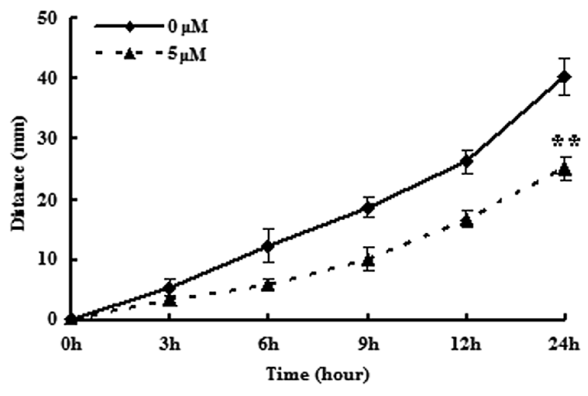

Figure 4. Disruption of AQP4 by PMA impairs the migratory ability of LN229 cells. (A) Western blot analysis indicating that expression of AQP4 was significantly reduced with PMA $(1$ and $5 \mu \mathrm{M})$ treatment for $24 \mathrm{~h}$ compared to the control cells. $\beta$-actin was used as a loading control. (B) Detection of the cytotoxicity of PMA to LN229 cells by MTT assay. A total of 3 doses (1, 5 and $10 \mu \mathrm{M})$ were used. MTT assay was performed after 24 h of PMA treatment. (C) Cell osmotic fragility was examined with or without PMA $(5 \mu \mathrm{M})$ treatment. (D) EGF-elicited chemotaxis assay of LN229 cells with or without PMA $(5 \mu \mathrm{M})$ treatment. (E) The scratch assay of LN229 cells with or without PMA $(5 \mu \mathrm{M})$ treatment. Data from three independent experiments are shown (** $\mathrm{p}<0.01$, two-way ANOVA analysis).

A

$\operatorname{scr} / \mathrm{LN} 229$

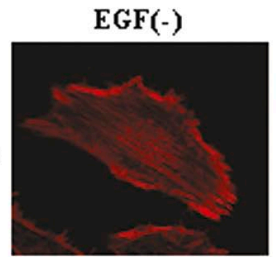

clone 2

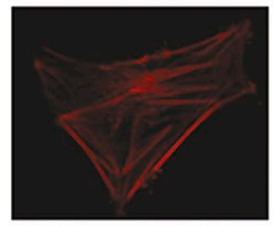

B

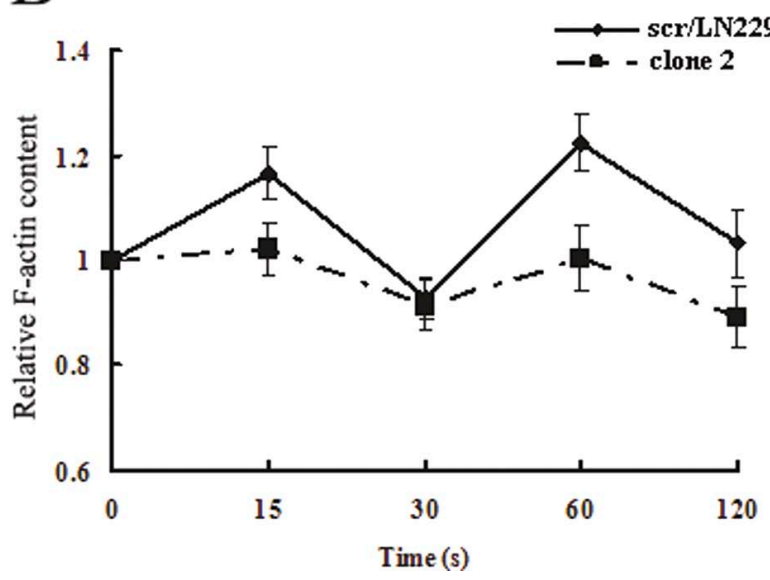

assays were subsequently performed to ascertain how AQP4 regulates LN229 cell adhesion. Previous studies found that the decreased ability of cell adhesion to a substrate was correlated with the decreased ability of cell migration $(11,28)$. In the present study, the ability of cells to adhere to a substrate was first determined, and the results are shown in Fig. 6A. We used a cell suspension to observe the number of cells which attached to a plastic substratum at 5, 15 and $30 \mathrm{~min}$, respectively. The results showed that the number of adherent siAQP4/LN229 clone 2 cells and PMA (5 $\mu \mathrm{M})$-treated cells were significantly decreased at 15 min compared to their control cells, respectively (Fig. 6A). In addition, the strength of cell anchorage to a plastic substratum was also determined using a detachment assay. This assay showed that siAQP4/ LN229 clone 2 cells detached after 6 min with trypsin treatment, whereas control cells detached after 13 min with trypsin treatment (Fig. 6B). Reduced intercellular adhesion followed by enhanced cell migration is an essential step in the progres-

Figure 5. Disruption of AQP4 by siRNA reduces F-actin polymerization in LN229 cells. (A) Cytoskeleton rearrangement in the scr/LN229 and siAQP4/LN229 clone 2 cells was imaged by F-actin fluorescence staining. Representative images from three repeated experiments are shown (x400). (B) Time course of relative F-actin content in scr/LN229 and siAQP4/LN29 clone 2 cells following $50 \mathrm{ng} / \mathrm{ml}$ of EGF stimulation. Data were obtained from three independent experiments. 

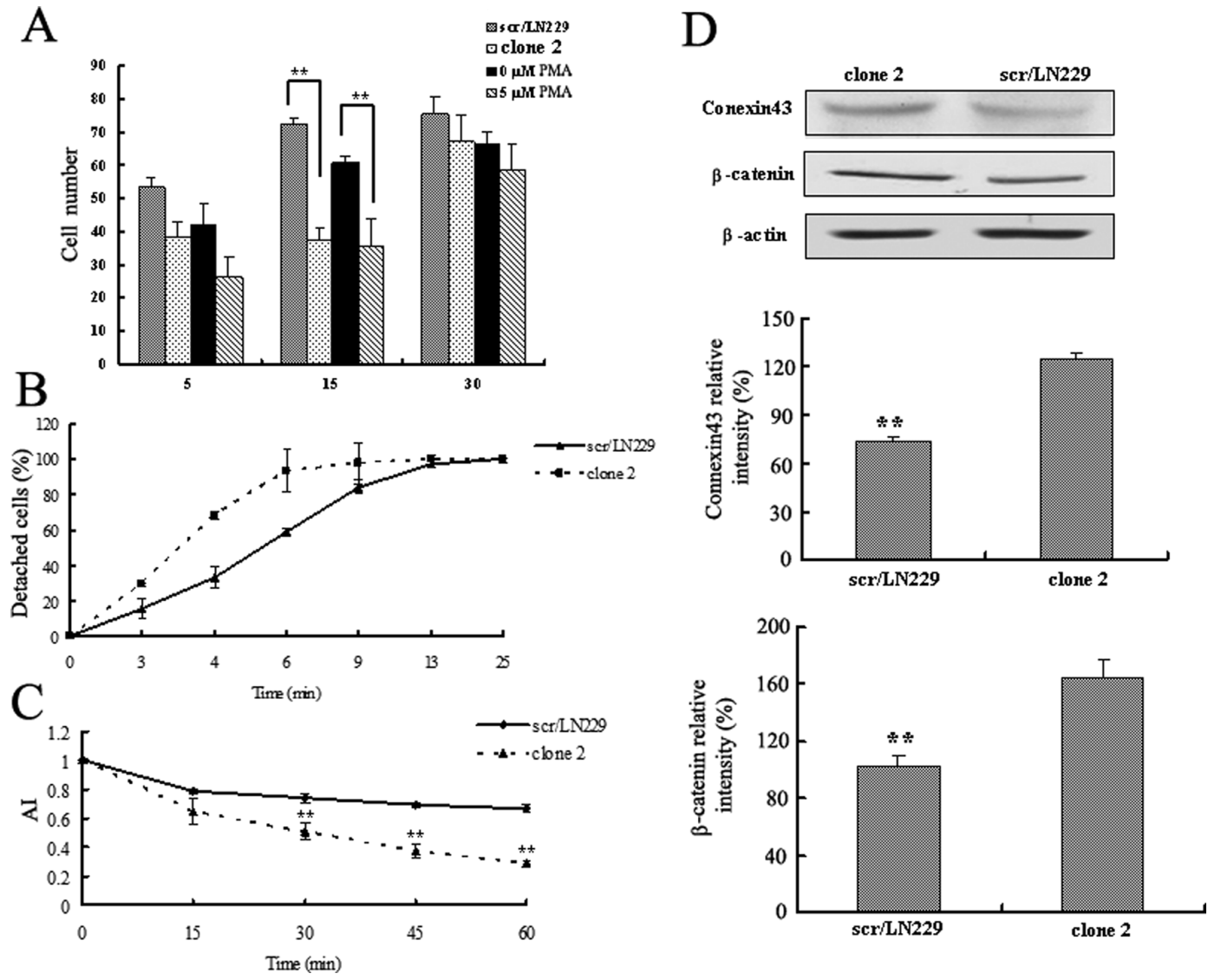

Figure 6. Reduction in AQP4 regulates LN229 cell adhesion. (A) Comparison of the substrate adhesion ability of scr/LN229, siAQP4/LN229 clone 2 and LN229 cells as well as PMA $(5 \mu \mathrm{M})$-treated cells at 5, 15 and $30 \mathrm{~min}$, respectively. Cell numbers were determined in 5 fields on every coverslip under microscopy (x200). A representative result from 3 independent experiments is shown. (B) The strength of cell (siAQP4/LN229 clone 2 and scr/LN2229 cells) attachment to substratum was estimated by the detachment assay. (C) Aggregation assay showing the aggregation index (AI) of the siAQP4/LN229 clone 2 and scr/LN2229 cells. (D) Western blotting was performed using either an anti-connexin 43 or an anti- $\beta$-catenin antibody in the scr/LN229 and siAQP4/ LN229 clone 2 cells. $\beta$-actin was used as a loading control. The intensity of the immunoreactivity in the Western blotting was calculated. Data were obtained from three independent experiments $(* * \mathrm{p}<0.01$, two-way ANOVA analysis).

sion from local malignancy to metastatic disease (29). In our study, an aggregation assay for detection of cell-cell adhesion was performed, and the result showed that the siAQP4/LN229 clone 2 cells exhibited greater ability to aggregate than the control cells (Fig. 6C).

$\beta$-catenin has been reported to promote actin reorganization and strengthening of cell-cell adhesion, and defects in its expression or function have been linked to tumor progression and metastasis (30). The gap junction protein connexin 43 which is expressed in most malignant gliomas is also essential for cell-cell adhesion and can function as an adhesion site which enhances cellular aggregation $(31,32)$. Therefore, we evaluated the expression of $\beta$-catenin and connexin 43 in LN229 cells with reduced AQP4. The Western blotting result showed that $\beta$-catenin and connexin 43 were both greatly increased in the siAQP4/LN229 clone 2 cells along with enhanced aggregation ability (Fig. 6C and D).

Reduction of AQP4 impairs LN229 cell invasion in vitro. The decrease in the migratory ability of cells usually leads to a decrease in the invasive ability (11). In the experiments described above, AQP4 was found to modulate the migration of LN229 cells. In the following experiment, we assessed the invasiveness of the cells with AQP4 downregulation in Matrigel Boyden chamber assays to determine whether AQP4 also affects cell invasion. EGF $(10 \mathrm{ng} / \mathrm{ml})$ was used as a chemoattractant to stimulate the cells to penetrate through the Matrigel and migrate through the filters. Matrigel contains laminin, heparin sulfate proteoglycan, entactin, and collagen type IV, which are extracellular matrix compositions of the normal brain and gliomas. The invasion assay showed prominent differences in invasive ability between the siAQP4/ LN229 clone 2 cells and the control ( $<<0.01$; Fig. 7A and B). The quantitative analysis of cell numbers revealed that the control cells had a 2.7 -fold higher rate of invasion than that of the siAQP4/LN229 clone 2 cells (Fig. 7B).

The matrix metalloproteinases (MMPs) play important roles in glioma invasion. Numerous studies have demonstrated high expression levels of MMP-2 and MMP-9 in high-grade gliomas (33). Considering the different invasive abilities of the siAQP4/LN229 clone 2 and the control cells in the above assay, we assessed the MMP-2 and the MMP-9 expression in the two groups of cells. We found that MMP-2 expression in siAQP4/LN229 clone 2 cells was significantly inhibited, while the MMP-9 expression remained almost unchanged (Fig. 7C). 
A

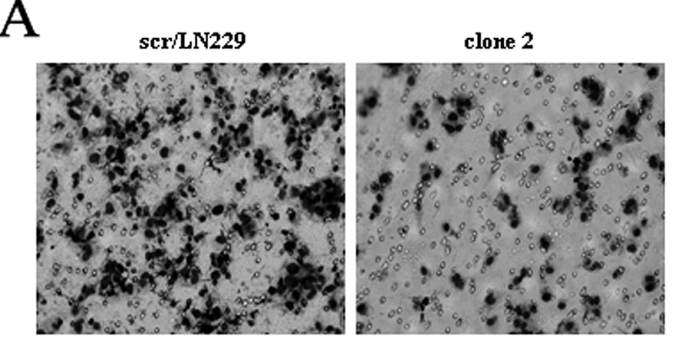

$\mathrm{C}$
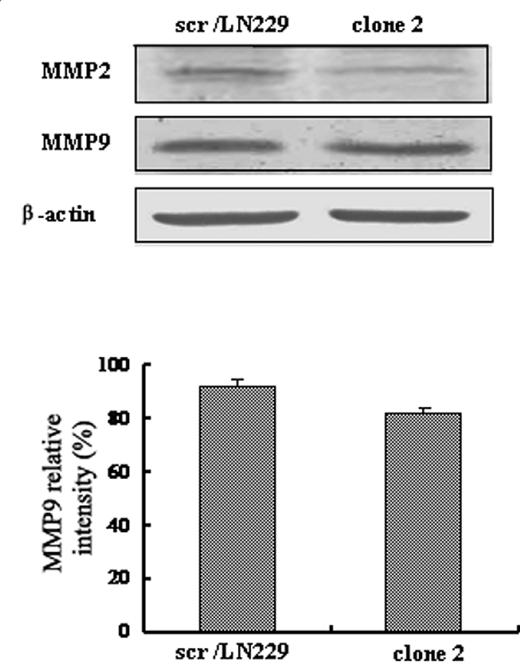
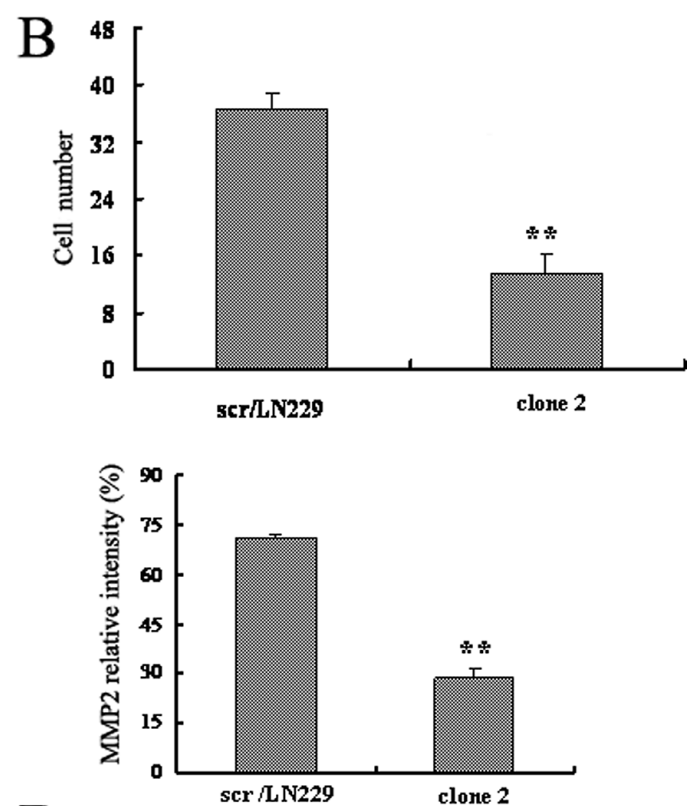

$\mathrm{D}$

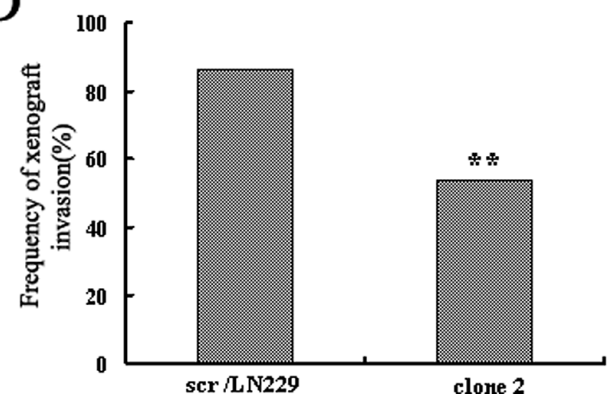

Figure 7. Disruption of AQP4 by siRNA impairs invasive ability in vitro and in vivo. (A) scr/LN229 and siAQP4/LN229 clone 2 cells invading through Matrigel-coated 8- $\mu \mathrm{m}$ pore size transwell inserts were evaluated as described in Material and methods. Images were captured at a magnification of x200. (B) Results of the in vitro Matrigel invasion assay. The number of invading cells was quantified by counting the stained cells in random fields of the membrane. All experiments were performed three times independently ("* $\mathrm{p}<0.01$, Student's t-test). (C) Western blotting of scr/LN229 and siAQP4/LN229 clone 2 cells was performed using either an anti-MMP-2 or an anti-MMP-9 antibody. $\beta$-actin was used as a loading control. The intensity of the immunoreactivity in the Western blotting was calculated. Data from three independent experiments are shown (** $\mathrm{p}<0.01$, two-way ANOVA analysis). (D) Frequency of xenograft invasion in the control $(\mathrm{n}=22)$ and the siAQP4/LN229 clone $2(\mathrm{n}=26)$ mouse groups $(* \mathrm{p}<0.05$, Chi-square test).

Reduction of AQP4 decreases LN229 cell invasion in vivo. To determine whether the in vitro assays described above have any bearing on tumor invasion in vivo, we applied an intracranial orthotopic human glioma model to validate the role of AQP4 in glioma invasion in vivo. $\mathrm{Nu} / \mathrm{Nu}$ mice $(\mathrm{n}=48)$ were used and divided into 2 groups. Each of the 2 groups was injected with siAQP4/LN229 clone 2 cells or scr/LN229 clone cells, respectively. The frequency of xenograft invasion in the siAQP4/LN229 clone 2 cell group [53.8\% (14/26)] was greatly decreased compared with the control group [86.4\% (19/22)] $(\mathrm{P}<0.05)$ (Fig. 7D).

\section{Discussion}

Research regarding AQP4 is not limited to brain edema. Increased AQP4 expression has been demonstrated in glioblastoma multiforme (GBM) together with loss of polarized expression around the vessels and AQP4 redistribution in glioma cells $(3,34,35)$. Auguste et al $(36)$ and Saadoun et al (37) found that AQP4 deletion in astroglial cells markedly impaired cell migration toward a stab wound in adult mouse brain and glial scar formation was markedly impaired in AQP4-null mice along with reduced migration of the reactive astroglia towards the site of brain injury $(36,37)$. Such results provide evidence of AQP4 involvement in cell migration during brain injury and suggest its involvement in brain tumor development.

Polarized cell migration is a tightly regulated process that occurs during tissue development, chemotaxis and wound healing. It is also highly associated with the infiltration and invasion of tumors (38). Here, we provide evidence for a new role of AQP4 in glioma migration and invasion. We found that AQP4 reduction slows glioma cell migration in cell chemotaxis and wound healing assays suggesting that AQP4 is involved in glioma cell migration. Meanwhile, AQP4 deletion did not affect cell proliferation in our study which was consistent with a previous report (39).

The formation and retraction of cell membrane protrusions at the leading edge of glioma cells are essential components of glioma migration and invasion (40). This change in cell shape is associated with rapid actin de-polymerization and re-polymerization, as well as altered ion fluxes at the front end 
of the cell, which produce rapid changes in intracellular osmolality driving transmembrane water flow $(40,41)$. It has been reported that $\mathrm{AQP} 4$ polarizes to lamellipodia and induces an increased number/size of lamellipodia in migrating cells, where rapid transmembrane water movements occur $(42,43)$. This is consistent with reports that ion channels and transporters playing a critical role in cell migration also polarize to the front end of migrating cells. Nicchia et al showed that AQP4 knockdown in astrocytes was associated with actin de-polymerization and dramatic cell morphological changes. Furthermore, a marked F-actin cytoskeleton rearrangement was also detected in AQP4 knockdown mouse brain where the cortical layer was completely replaced by fibers in a star-like organization (44). Moreover, AQP4 was found to interact with $\alpha$-syntrophin which is a member of the dystrophin-dystroglycan complex. This complex includes molecules which link the actin cytoskeleton and $\beta$-dystroglycan (45). These findings strongly indicate an involvement of the AQP4 protein in the alteration of cell cytoskeleton (46). In the present study, we showed that the reduction of AQP4 impairs glioma cell migration by reducing F-actin polymerization (Fig. 5B). It was proposed that, as a consequence of actin polymerization/ de-polymerization and transmembrane ionic fluxes, the cytoplasm adjacent to the leading edge of migrating cells undergoes rapid changes in osmolality. AQP4 may thus facilitate osmotic water flow across the plasma membrane in cell protrusions during glioma cell migration.

Cell-cell adhesiveness is generally reduced in human cancers. Reduced intercellular adhesion followed by cell invasion is an essential step in the progression from local malignancy to metastatic disease (46). The control and maintenance of normal intercellular adhesion are regulated by the cadherin-catenin cell adhesion complex. $\beta$-catenin and connexin 43 , the multifunctional proteins commonly found in excess levels in certain types of cancer, are key players in both calcium-dependent intercellular adhesion events and nuclear gene expression via the Wnt pathway $(29,47)$. In gliomas, these proteins are deregulated leading to an accumulation of protein that can cause both the loss of cell-cell adhesion and increased transcription of target genes (48). Our study showed that the cell-cell adhesion ability of glioma cells was greatly increased after reduction of AQP4, and the adhesion ability to the extracellular matrix was also impaired. The expression of $\beta$-catenin and connexin 43 was increased in AQP4-downregulated LN229 cells consistent with their enhanced cell-cell adhesion. We speculate that such a phenomenon was probably due to the impaired invasive ability of AQP4-reduced LN229 cells.

Similar to migration and adhesion, invasion through the extracellular matrix is an important step in tumor invasion (49). Our Matrigel invasion assay demonstrated that reduction of AQP4 led to a decrease in invasiveness of the LN229 cells. Furthermore, as demonstrated by the detection of invasion in vivo, the frequency of xenograft invasion in mice decreased significantly after AQP4 downregulation compared with the control, consistent with the invasion assay results in vitro.

Glioma invasion into normal brain regions involves the disruption of the ECM and subsequent penetration into adjacent brain structures. This process is accomplished by the secretion of MMPs by tumor cells (50). The MMPs are a family of 20 enzymes that have the ability to degrade the ECM. Their activity results in the breakdown of connective tissue barriers such as collagens, laminins, fibronectin, vitronectin and HS proteoglycans (51). Elevated levels of MMPs have been reported to be correlated with tumor cell invasiveness of gliomas and are believed to play an important role in cellular invasion and metastasis $(52,53)$. In particular, MMP-2 and MMP-9 levels increase with tumor progression in gliomas and are thus known as key enzymes in invasion (54). Numerous studies have demonstrated that elevated MMP-2 levels correlate with increased invasiveness in gliomas $(55,56)$ and MMP-2 expression has frequently been correlated with malignant phenotypes $(56,57)$. In this study, our results revealed that the expression of MMP-2 was significantly decreased in the siAQP4/LN229 clone 2 cells while MMP-9 was invariable. The inhibition of MMP-2 expression by reduction of AQP4 explains the inhibitory effect of AQP4 on glioma cell invasion.

Understanding of the molecular mechanisms involved in glioma invasion is critical for the developement of novel therapeutic strategies or treatments since major therapeutic approaches such as surgery and radiotherapy are ineffective without blocking glioma invasion. In the present study, we discovered a new function for AQP4 in glioma cells. This property of AQP4 appears unrelated to the well-established role of AQP4 in brain edema formation and absorption (58), and suggests novel therapeutic possibilities. Screening for specific small-molecular inhibitors of AQP4 may provide new insights for the design of novel mechanism-based therapies for glioma and may shed light on an important biological aspect of glioma progression. At present, the AQP4 antibody has been suggested for the treatment and diagnosis of neuromyelitis optica, an inflammatory disease mainly affecting the optic nerve and spinal cord $(59,60)$. Hopefully, improved chemotherapy targeting AQP4 may be available to patients with glioma in the next few years.

\section{Acknowledgements}

This work was supported by the China 973 (2009CB521705) and 863 programs (2006AA02A249) (2007AA021802), the National Scientific Foundation of China (30700253) (30800355), Key Program of the National Scientific Foundation of China (30930038), the China Program of the Changjiang Scholar and Innovative Research Team in the University (IRT0743).

\section{References}

1. Zador Z, Bloch O, Yao X and Manley GT: Aquaporins: role in cerebral edema and brain water balance. Prog Brain Res 161: 185-194, 2007.

2. Yang JH, Shi YF, Cheng Q and Deng L: Expression and localization of aquaporin-5 in epithelial ovarian tumors. Gynecol Oncol 100: 294-299, 2006.

3. Warth A, Mittelbronn M and Wolburg H: Redistribution of the water channel protein aquaporin- 4 and the $\mathrm{K}^{+}$channel protein Kir4.1 differs in low- and high-grade human brain tumors. Acta Neuropathol 109: 418-426, 2005.

4. Verkman AS: More than just water channels: unexpected cellular roles of aquaporins. J Cell Sci 118: 3225-3232, 2005.

5. Venero JL, Vizuete ML, Machado A and Cano J: Aquaporins in the central nervous system. Prog Neurobiol 63: 321-336, 2001. 
6. Senger D, Cairncross JG and Forsyth PA: Long-term survivors of glioblastoma: statistical aberration or important unrecognized molecular subtype? Cancer J 9: 214-221, 2003.

7. Hu $J$ and Verkman AS: Increased migration and metastatic potential of tumor cells expressing aquaporin water channels. FASEB J 20: 1892-1894, 2006.

8. Hoque MO, Soria JC, Woo J, Lee T, Lee J, Jang SJ, Upadhyay S, Trink B, Monitto C, Desmaze C, Mao L, Sidransky D and Moon C: Aquaporin 1 is overexpressed in lung cancer and stimulates NIH-3T3 cell proliferation and anchorage-independent growth. Am J Pathol 168: 1345-1353, 2006.

9. Giese A and Westphal M: Glioma invasion in the central nervous system. Neurosurgery 39: 235-250, 1996.

10. Badaut J, Lasbennes F, Magistretti PJ and Regli L: Aquaporins in brain: distribution, physiology, and pathophysiology. J Cereb Blood Flow Metab 22: 367-378, 2002.

11. Zhang B, Gu F, She C, Guo H, Li W, Niu R, Fu L, Zhang N and Ma Y: Reduction of Akt2 inhibits migration and invasion of glioma cells. Int J Cancer 125: 585-595, 2009.

12. Gu F, Hata R, Toku K, Yang L, Ma YJ, Maeda N, Sakanaka M and Tanaka J: Testosterone up-regulates aquaporin-4 expression in cultured astrocytes. J Neurosci Res 72: 709-715, 2003.

13. Sun R, Gao P, Chen L, Ma D, Wang J, Oppenheim JJ and Zhang N: Protein kinase $\mathrm{C}$ zeta is required for epidermal growth factor-induced chemotaxis of human breast cancer cells. Cancer Res 65: 1433-1441, 2005.

14. Grille SJ, Bellacosa A, Upson J, Klein-Szanto AJ, van Roy F, Lee-Kwon W, Donowitz M, Tsichlis PN and Larue L: The protein kinase Akt induces epithelial mesenchymal transition and promotes enhanced motility and invasiveness of squamous cell carcinoma lines. Cancer Res 63: 2172-2178, 2003.

15. Asano K, Duntsch CD, Zhou Q, Weimar JD, Bordelon D, Robertson JH and Pourmotabbed T: Correlation of N-cadherin expression in high grade gliomas with tissue invasion. J Neurooncol 70: 3-15, 2004.

16. Takino T, Nakada M, Miyamori H, Yamashita J, Yamada KM and Sato H: CrkI adapter protein modulates cell migration and invasion in glioblastoma. Cancer Res 63: 2335-2337, 2003.

17. Nicchia GP, Frigeri A, Liuzzi GM and Svelto M: Inhibition of aquaporin-4 expression in astrocytes by RNAi determines alteration in cell morphology, growth, and water transport and induces changes in ischemia-related genes. FASEB J 17: $1508-1510,2003$

18. Papadopoulos MC, Saadoun S and Verkman AS: Aquaporins and cell migration (Review). Pflugers Arch 456: 693-700, 2008.

19. Dancey JE and Freidlin B: Targeting epidermal growth factor receptor - are we missing the mark? Lancet 362: 62-64, 2003.

20. Ji C, Cao C, Lu S, Kivlin R, Amaral A, Kouttab N, Yang H, Chu W, Bi Z, Di W and Wan Y: Curcumin attenuates EGF-induced AQP3 up-regulation and cell migration in human ovarian cancer cells. Cancer Chemother Pharmacol 62: 857-865, 2008.

21. Etienne-Manneville S and Hall A: Integrin-mediated activation of Cdc 42 controls cell polarity in migrating astrocytes through PKCzeta. Cell 106: 489-498, 2001.

22. Nakahama K, Nagano M, Fujioka A, Shinoda K and Sasaki H: Effect of TPA on aquaporin 4 mRNA expression in cultured rat astrocytes. Glia 25: 240-246, 1999.

23. Doerr ME and Jones JI: The roles of integrins and extracellular matrix proteins in the insulin-like growth factor I-stimulated chemotaxis of human breast cancer cells. J Biol Chem 271: 2443-2447, 1996.

24. Zigmond SH, Joyce M, Borleis J, Bokoch GM and Devreotes PN: Regulation of actin polymerization in cell-free systems by GTPgammaS and Cdc42. J Cell Biol 138: 363-374, 1997.

25. Ananthakrishnan R and Ehrlicher A: The forces behind cell movement. Int J Biol Sci 3: 303-317, 2007.

26. Chen L, Janetopoulos C, Huang YE, Iijima M, Borleis J and Devreotes PN: Two phases of actin polymerization display different dependencies on $\mathrm{PI}(3,4,5) \mathrm{P} 3$ accumulation and have unique roles during chemotaxis. Mol Biol Cell 14: 5028-5037, 2003.

27. Hirohashi S and Kanai Y: Cell adhesion system and human cancer morphogenesis. Cancer Sci 94: 575-581, 2003.

28. Guo H, Gu F, Li W, Zhang B, Niu R, Fu L, Zhang N and Ma Y: Reduction of protein kinase $\mathrm{C}$ zeta inhibits migration and invasion of human glioblastoma cells. J Neurochem 109 203-213, 2009.

29. Polakis P: Wnt signaling and cancer. Genes Dev 14: 1837-1851, 2000.
30. Barami K, Lewis-Tuffin L and Anastasiadis PZ: The role of cadherins and catenins in gliomagenesis. Neurosurg Focus 21: E13, 2006.

31. Huang RP, Hossain MZ, Sehgal A and Boynton AL: Reduced connexin43 expression in high-grade human brain glioma cells. J Surg Oncol 70: 21-24, 1999.

32. Zhang W, Couldwell WT, Simard MF, Song H, Lin JH and Nedergaard M: Direct gap junction communication between malignant glioma cells and astrocytes. Cancer Res 59: 1994-2003, 1999.

33. Uhm JH, Dooley NP, Villemure JG and Yong VW: Mechanisms of glioma invasion: role of matrix-metalloproteinases. Can J Neurol Sci 24: 3-15, 1997.

34. Warth A, Kroger S and Wolburg H: Redistribution of aquaporin-4 in human glioblastoma correlates with loss of agrin immunoreactivity from brain capillary basal laminae. Acta Neuropathol 107: 311-318, 2004

35. Warth A, Simon P, Capper D, Goeppert B, Tabatabai G, Herzog H, Dietz K, Stubenvoll F, Ajaaj R, Becker R, Weller M, Meyermann R, Wolburg H and Mittelbronn M: Expression pattern of the water channel aquaporin-4 in human gliomas is associated with blood-brain barrier disturbance but not with patient survival. J Neurosci Res 85: 1336-1346, 2007.

36. Auguste KI, Jin S, Uchida K, Yan D, Manley GT, Papadopoulos MC and Verkman AS: Greatly impaired migration of implanted aquaporin-4-deficient astroglial cells in mouse brain toward a site of injury. FASEB J 21: 108-116, 2007.

37. Saadoun S, Papadopoulos MC, Watanabe H, Yan D, Manley GT and Verkman AS: Involvement of aquaporin-4 in astroglial cell migration and glial scar formation. J Cell Sci 118: 5691-5698, 2005.

38. Noritake J, Watanabe T, Sato K, Wang S and Kaibuchi K: IQGAP1: a key regulator of adhesion and migration. J Cell Sci 118: 2085-2092, 2005.

39. McCoy E and Sontheimer H: Expression and function of water channels (aquaporins) in migrating malignant astrocytes. Glia 55: 1034-1043, 2007.

40. Small JV, Stradal T, Vignal E and Rottner K: The lamellipodium: where motility begins. Trends Cell Biol 12: 112-120, 2002.

41. Schwab A: Function and spatial distribution of ion channels and transporters in cell migration. Am J Physiol Renal Physiol 280: F739-F747, 2001

42. Saadoun S, Papadopoulos MC, Hara-Chikuma M and Verkman AS: Impairment of angiogenesis and cell migration by targeted aquaporin-1 gene disruption. Nature 434: 786-792, 2005.

43. Hara-Chikuma M and Verkman AS: Aquaporin-1 facilitates epithelial cell migration in kidney proximal tubule. J Am Soc Nephrol 17: 39-45, 2006.

44. Nicchia GP, Srinivas M, Li W, Brosnan CF, Frigeri A and Spray DC: New possible roles for aquaporin-4 in astrocytes: cell cytoskeleton and functional relationship with connexin 43 . FASEB J 19: 1674-1676, 2005.

45. Neely JD, Amiry-Moghaddam M, Ottersen OP, Froehner SC, Agre P and Adams ME: Syntrophin-dependent expression and localization of Aquaporin-4 water channel protein. Proc Natl Acad Sci USA 98: 14108-14113, 2001.

46. Ding T, Gu F, Fu L and Ma YJ: Aquaporin-4 in glioma invasion and an analysis of molecular mechanisms. J Clin Neurosci 17: 1359-1361, 2010.

47. Gottardi CJ and Gumbiner BM: Distinct molecular forms of betacatenin are targeted to adhesive or transcriptional complexes. J Cell Biol 167: 339-349, 2004

48. Brembeck FH, Rosario M and Birchmeier W: Balancing cell adhesion and Wnt signaling, the key role of beta-catenin. Curr Opin Genet Dev 16: 51-59, 2006.

49. Amos S, Mut M, diPierro CG, Carpenter JE, Xiao A, Kohutek ZA, Redpath GT, Zhao Y, Wang J, Shaffrey ME and Hussaini IM: Protein kinase C-alpha-mediated regulation of low-density lipoprotein receptor-related protein and urokinase increases astrocytoma invasion. Cancer Res 67: 10241-10251, 2007.

50. Rao JS: Molecular mechanisms of glioma invasiveness: the role of proteases. Nat Rev Cancer 3: 489-501, 2003

51. Stamenkovic I: Extracellular matrix remodelling: the role of matrix metalloproteinases. J Pathol 200: 448-464, 2003.

52. Björklund $\mathrm{M}$ and Koivunen E: Gelatinase-mediated migration and invasion of cancer cells. Biochim Biophys Acta 1755: 37-69, 2005.

53. Wild-Bode C, Weller M and Wick W: Molecular determinants of glioma cell migration and invasion. J Neurosurg 94: 978-984, 2001. 
54. Forsyth PA, Wong H, Laing TD, Rewcastle NB, Morris DG, Muzik H, Leco KJ, Johnston RN, Brasher PM, Sutherland G and Edwards DR: Gelatinase-A (MMP-2), gelatinase-B (MMP-9) and membrane type matrix metalloproteinase-1 (MT1-MMP) are involved in different aspects of the pathophysiology of malignant gliomas. Br J Cancer 79: 1828-1835, 1999.

55. Lampert K, Machein U, Machein MR, Conca W, Peter HH and Volk B: Expression of matrix metalloproteinases and their tissue inhibitors in human brain tumors. Am J Pathol 153: 429-437, 1998.

56. Sawaya RE, Yamamoto M, Gokaslan ZL, Wang SW, Mohanam S, Fuller GN, McCutcheon IE, Stetler-Stevenson WG, Nicolson GL and Rao JS: Expression and localization of $72 \mathrm{kDa}$ type IV collagenase (MMP-2) in human malignant gliomas in vivo. Clin Exp Metastasis 14: 35-42, 1996.
57. Corcoran ML, Hewitt RE, Kleiner DE Jr and StetlerStevenson WG: MMP-2: expression, activation and inhibition. Enzyme Protein 49: 7-19, 1996.

58 Tomas-Camardiel M, Venero JL, de Pablos RM, Rite I, Machado A and Cano J: In vivo expression of aquaporin-4 by reactive microglia. J Neurochem 91: 891-899, 2004.

59. Misu T, Takahashi T, Nakashima I, Fujihara K and Itoyama Y: [Neuromyelitis optica and anti-aquaporin 4 antibody - distinct from multiple sclerosis]. Rinsho Byori 57: 262-270, 2009.

60. Jarius S and Wildemann B: AQP4 antibodies in neuromyelitis optica: diagnostic and pathogenetic relevance. Nat Rev Neurol 6: 383-392, 2010 